\title{
Risk assessment of renal cortical scarring with urinary tract infection by clinical features and ultrasonography
}

\author{
M T Christian, J H McColl, J R MacKenzie, T J Beattie
}

\begin{abstract}
Aims-To address some of the issues in the ongoing debate over the optimal diagnostic imaging following childhood urinary tract infection (UTI), by determining the risk of missing renal cortical scarring which would be detected on a technetium99m dimercaptosuccinic acid (DMSA) gold standard if ultrasound alone were used, factoring for clinical features (upper or lower tract), UTI recurrence, and age group (infants, preschool, or school age). Methods-Details of UTI clinical features and recurrence were recorded for 990 children with a proven UTI, and their DMSA and ultrasound results were compared for each kidney.

Results-The risks of missing DMSA scarring varied between $0.4 \%$ (school age children with solitary lower tract UTI) and $11.1 \%$ (infants with recurrent upper tract UTI).

Conclusions-UTI clinical features are important in assessing the need for DMSA imaging. Current UK imaging guidelines are endorsed, although preschool children with solitary lower tract UTI remain a controversial group and more attention needs to focused on children with recurrent UTI.

(Arch Dis Child 2000;82:376-380)
\end{abstract}

Keywords: urinary tract infection; DMSA; ultrasonography; renal cortical scarring

Urinary tract infection (UTI) is one of the most common bacterial diseases of childhood, with a reported prevalence in one study of $8.4 \%$ in girls and $1.7 \%$ in boys by the age of 7 years. ${ }^{1}$ Diagnostic imaging of the urinary tract in children with a history of UTI has been accepted practice since the 1960 s, and seminal studies from this decade ${ }^{2}$ showed a high incidence of abnormalities, particularly chronic pyelonephritis and vesicoureteric reflux, showing that the condition was far from benign. In a more recent study, renal parenchymal scarring was found in $12 \%{ }^{3}$ and vesicoureteric reflux in $17 \%^{4}$ of kidneys in a large cohort of such children.

Most children with UTI have an excellent prognosis but there is an appreciable risk of long term complications in those with obstructive malformations and dilated vesicoureteric reflux, particularly if there has been a delay in diagnosis and/or inadequate treatment. A long term prospective study has shown a $10 \%$ risk of end stage renal failure and a $23 \%$ risk of hyper- tension in a group of 30 patients with renal scarring diagnosed 27 years earlier. ${ }^{5}$ Studies in adult women have shown an association between childhood renal scarring and pregnancies complicated by bacteriuria, acute pyelonephritis, hypertension, pre-eclampsia, and interventional deliveries. ${ }^{5-7}$

Imaging protocols in children with UTI continue to be a subject of debate. ${ }^{8}$ Table 1 shows the current practice guidelines published by the British Paediatric Association (BPA). ${ }^{9}$ The advantages of ultrasound include the lack of ionising radiation, general availability, and patient acceptability. These must be balanced against the disadvantages of operator dependence and lack of functional information provided. A recent paper detailing a systematic approach to ultrasound specifically for the detection of renal scarring shows promise as a means to improve ultrasound sensitivity. ${ }^{10}$ In the context of UTI, ultrasound is generally the first line investigation which allows identification of structural abnormalities, both congenital and acquired. The sensitivity of ultrasound in detecting renal cortical scarring however, is not high and renal scintigraphy by technetium99m dimercaptosuccinic acid (DMSA) scanning is now generally accepted as the most sensitive form of renal parenchymal imaging. ${ }^{11}$

As renal scintigraphy is an invasive means of renal imaging, there is a need to selectively screen which children should undergo this investigation. Taking the lead from a research suggestion in a recent report of a working group that formulated guidelines for the management of acute UTI in childhood, ${ }^{8}$ the aim of the study was to determine in a large cohort of children, the risk of missing renal cortical scarring detected by a DMSA gold standard if ultrasound alone were used, and to factor this risk for clinical features, UTI recurrence, and age group corresponding to the BPA practice guidelines (infants, preschool, or school age).

Table 1 British Paediatric Association practice guidelines*

\begin{tabular}{ll}
\hline Up to 1 year & $\begin{array}{l}\text { Ultrasound of the urinary system, } \\
\text { contrast cystography, DMSA } \dagger\end{array}$ \\
1-4 years inclusive & $\begin{array}{l}\text { Ultrasound of the urinary system, } \\
\text { DMSA } \neq\end{array}$ \\
(a) Those with "cystitis"/lower tract \\
symptoms-ultrasound +/- \\
abdominal $x$ ray \\
(b) Those with systemic \\
symptoms-ultrasound, DMSA +/- \\
abdominal $x$ ray
\end{tabular}

*Pilling and Postlethwaite.

+Some use this plan of investigation up to 2 years.

$\ddagger$ Some would advise selective use of DMSA as for 5 years and over. 


\section{Subjects and methods}

A database was constructed of all children referred to the renal outpatient clinic at the Royal Hospital for Sick Children, Yorkhill, Glasgow, and those children admitted to the same hospital who were under, or referred to the renal service as inpatients, between 1990 and 1996 inclusive. Only children with bacteriologically proven UTI were included, as evidenced by the bacterial culture report or documentation in the referral letter that the infection had been confirmed (on a clean catch or suprapubic bladder aspirate in infants or a mid stream specimen in older children). Children who were known to have a preexistent urinary tract abnormality or who had undergone previous renal imaging (rereferrals) were excluded. Only children who underwent both ultrasound and DMSA were included. To make a valid comparison between the two modalities, all records where the imaging took place more than 90 days apart were excluded. To exclude those children with possible transient DMSA changes, all children whose DMSA was carried out within 30 days of the original consultation were also excluded.

For each child basic demographic details were included as well as the date of first consultation, the date and results of DMSA and ultrasound imaging for each kidney, whether the UTI was single or recurrent, and the clinical features at presentation. These included one or more of the following: fever, vomiting, lethargy, general systemic upset, loin pain, non-specific abdominal pain, frequency, dysuria, incontinence, frank haematuria, or urgency. Any child in whom the symptoms recorded in the case record or referral letter were incomplete or absent, was excluded. The clinical features were used to classify the UTI into upper tract or lower tract. Any child with one or more of the first five symptoms listed was said to have had an upper tract UTI.

The ultrasound examinations were carried out with an Acuson 128 XP10 system (Mountain View, California) with a $5 \mathrm{MHz}$ probe. Renal images were obtained in the supine and prone positions and the bladder was assessed. Scanning was performed or supervised by an experienced consultant paediatric radiologist. Abnormal reports were classified for each kidney as: scarring; pelvic-ureteric dilatation; hydronephrosis; large, small, or no kidney; duplex kidney; renal swelling; increased or decreased echogenicity; prominence of the pyramids; loss of corticomedullary differentiation; or renal cyst(s).

${ }^{99 \mathrm{~m}}$ Tc DMSA scanning was carried out two hours after intravenous administration of a weight related dose of isotope up to a maximum of $80 \mathrm{MBq}{ }^{99 \mathrm{~m}} \mathrm{Tc}$ DMSA. Images were obtained using a high resolution collimator, with the child in the posterior, right posterior oblique, and left posterior oblique position. Analogue images were used for reporting which was carried out by an experienced consultant paediatric radiologist. The abnormal imaging was specified as: scarring; focal or generalised photon deficient areas; large, small, or no kidney; possible duplex kidney; or abnor- mal differential function. Cortical scarring was defined as a defect in the normal reniform outline. A photon deficient area was defined as a focal or diffuse area of reduced uptake of the radionuclide with preservation of the normal reniform outline. Normal differential function was defined as a disparity no greater than $45 \%: 55 \% .{ }^{12}$ Where there was abnormal differential function, only the kidney with the low differential function was recorded as abnormal if there were no other abnormalities.

The children were divided into age groups according to their age at the time of ultrasound examination. The age groups chosen corresponded to those in the BPA guidelines: infants, preschool (1-4 years inclusive), and school age (5 years and above). These age groups were further subdivided into those with upper or lower tract UTI and those with recurrent or solitary UTI.

Comparing DMSA and ultrasound results for each kidney, the risk of missing abnormalities and/or cortical scarring was calculated as the percentage of kidneys with normal ultrasound in which abnormalities and/or scarring were detected when using DMSA as a gold standard. The results obtained were factored for age group, clinical features, and recurrence. Similarly the sensitivity of ultrasound was calculated as the percentage of kidneys with DMSA abnormalities and/or scarring that were correctly identified as abnormal and/or scarred on ultrasound.

Results are reported as percentages, with approximate $95 \%$ confidence intervals, derived using a normal approximation, in parentheses. Significance was calculated using standard errors of proportions. Tests of association between categorical variables were carried out using a $\chi^{2}$ test.

\section{Results}

The database comprised 990 children of whom $250(25 \%)$ were boys and $740(75 \%)$ girls. A total of 404 children had upper tract UTI and 586 lower tract UTI. Table 2 shows the proportions within individual age groups. In total, $172(17.4 \%(14.8-20.0 \%))$ children had abnormal DMSA findings in one or both kidneys and $84(8.5 \%(6.7-10.3 \%))$ children had cortical scarring on DMSA in one or both kidneys (table 3 ). Thirty children had bilateral DMSA abnormalities and eight had bilateral scarring. There were significantly more children with abnormal DMSA and DMSA scarring in those who had upper tract UTI compared with those who had lower tract UTI, both overall and in individual age groups $\left(\mathrm{p}<0.001 ; \chi^{2}\right.$ test). Similarly there were significantly more children with abnormal

Table 2 Breakdown of upper and lower UTI

\begin{tabular}{lllllll}
\hline & \multicolumn{2}{c}{ Upper UTI } & & \multicolumn{2}{c}{ Lower UTI } \\
\cline { 3 - 4 } \cline { 7 - 7 } & Total (boys) & $n$ & $\%$ & & $n$ & $\%$ \\
\hline Infants & $38(25)$ & 35 & 92 & & 3 & 8 \\
Preschool & $570(140)$ & 242 & 42 & & 328 & 58 \\
School age & $382(85)$ & 127 & 33 & & 255 & 67 \\
Total & $990(250)$ & 404 & 41 & & 586 & 59 \\
\hline
\end{tabular}


Table 3 Rates of DMSA abnormalities and scarring

\begin{tabular}{|c|c|c|c|c|c|c|c|c|c|c|c|c|}
\hline & \multicolumn{6}{|c|}{ Abnormal DMSA } & \multicolumn{6}{|c|}{$D M S A$ scarring } \\
\hline & \multicolumn{2}{|c|}{ Total } & \multicolumn{2}{|c|}{ Upper } & \multicolumn{2}{|c|}{ Lower } & \multicolumn{2}{|c|}{ Total } & \multicolumn{2}{|c|}{ Upper } & \multicolumn{2}{|c|}{ Lower } \\
\hline & $\mathrm{n}$ & $\%$ & $\bar{n}$ & $\%$ & $\mathrm{n}$ & $\%$ & $\mathrm{n}$ & $\%$ & $\mathrm{n}$ & $\%$ & $\mathrm{n}$ & $\%$ \\
\hline Infants & 9 & 24 & 9 & 26 & 0 & 0 & 6 & 16 & 6 & 17 & 0 & 0 \\
\hline Preschool & 96 & 17 & 58 & 24 & 38 & 12 & 45 & 8 & 27 & 11 & 18 & 5 \\
\hline School age & 67 & 18 & 37 & 29 & 30 & 12 & 33 & 9 & 21 & 17 & 12 & 5 \\
\hline Totals & 172 & 17 & 104 & 26 & 68 & 12 & 84 & 8 & 54 & 13 & 30 & 5 \\
\hline
\end{tabular}

Table 4 DMSA abnormalities and normal ultrasound scans

\begin{tabular}{llll}
\hline Abnormality & Total kidneys & $\begin{array}{l}\text { Kidneys with } \\
\text { normal ultrasound }\end{array}$ & $\begin{array}{l}\text { \% with normal } \\
\text { ultrasound }\end{array}$ \\
\hline Scarring & 92 & 52 & 57 \\
Focal photon deficiency & 69 & 46 & 67 \\
Abnormal differential function & 75 & 42 & 56 \\
?Duplex & 35 & 20 & 57 \\
Large kidney & 19 & 9 & 47 \\
Small kidney & 31 & 12 & 39 \\
Generalised photon deficiency & 18 & 13 & 72 \\
Other & 4 & 2 & 50 \\
No kidney & 1 & 0 & 0 \\
\hline
\end{tabular}

DMSA and DMSA scarring in those who had recurrent UTI ( $p<0.001 ; \chi^{2}$ test). There were no significant differences in the percentage of children with abnormal DMSA or DMSA scarring between different age groups.

Of 204 kidneys where there was one or more DMSA abnormalities, the ultrasound was reported as normal in $127(62 \%)$. Table 4 shows the DMSA abnormalities detected, along with details of the corresponding ultrasound findings.

The sensitivity of ultrasound for the detection of any DMSA abnormality was $37.7 \%$ (34.7-40.8\%) overall, and varied from $16.0 \%$ in preschool children with solitary lower tract UTI to $58.6 \%$ in school age children with recurrent lower tract UTI. The sensitivity of ultrasound for the detection of renal cortical scarring was $21.7 \%(19.2-24.3 \%)$ overall, and varied from $7.7 \%$ in preschool children with solitary upper tract UTI to $33.3 \%$ in school age children with recurrent lower tract UTI. For both the detection of scarring and any DMSA abnormality, ultrasound was significantly $(p<0.05)$ less sensitive in preschool age children than in school age children, but the

Table 5 Risk of missing DMSA abnormalities on ultrasound

\begin{tabular}{llllll}
\hline & \multirow{2}{*}{ Upper } & & & \multicolumn{2}{l}{ Lower } \\
\cline { 2 - 3 } \cline { 5 - 6 } & Recurrent & Solitary & & Recurrent & Solitary \\
\hline Infants & $22.2(0-48.0)$ & $11.8(3.6-19.9)$ & & N/A & N/A \\
Preschool & $15.3(9.3-21.4)$ & $8.8(5.8-11.8)$ & & $4.0(1.4-6.7)$ & $4.9(2.9-7.0)$ \\
School age & $16.1(9.8-22.3)$ & $6.2(1.9-10.5)$ & & $5.0(2.4-7.6)$ & $2.2(0.3-4.0)$ \\
\hline
\end{tabular}

Figures are for individual kidneys and are expressed as percentages with $95 \%$ confidence intervals in parentheses.

N/A, figures not available.

Table 6 Risk of missing cortical scarring on ultrasound

\begin{tabular}{llllll}
\hline & \multicolumn{1}{l}{ Upper } & & & \multicolumn{2}{l}{ Lower } \\
\cline { 2 - 3 } \cline { 6 - 6 } \cline { 5 - 6 } & Recurrent & Solitary & & Recurrent & Solitary \\
\hline Infants & $11.2(0-30.6)$ & $5.9(0-11.8)$ & & N/A & N/A \\
Preschool & $8.9(4.1-13.7)$ & $2.4(0.8-4.0)$ & & $2.5(0.4-4.6)$ & $1.2(0.2-2.2)$ \\
School age & $8.9(4.1-13.8)$ & $4.4(0.8-8.1)$ & & $1.2(0-2.6)$ & $0.4(0-1.3)$ \\
\hline
\end{tabular}

Figures are for individual kidneys and are expressed as percentages with $95 \%$ confidence intervals in parentheses.

N/A, figures not available. decreased sensitivity in infants compared to preschool age children did not reach statistical significance.

For all children in the study, the risk of missing DMSA abnormalities if ultrasound was the sole imaging procedure would have been $6.9 \%$ $(5.8-8.0 \%)$ and the risk of missing cortical scarring would have been $2.8 \%(2.1-3.6 \%)$. However, factoring for the three variables of age group, clinical features, and recurrence, the risks of missing DMSA abnormalities would have varied between $2.2 \%$ and $22.0 \%$ (table 5 ), and the risks of missing cortical scarring would have varied between $0.4 \%$ and $11.1 \%$ (table 6 ).

The characteristics of the kidneys where there was scarring on DMSA but a normal ultrasound $(\mathrm{S} / \mathrm{N})$ were compared with those where there was scarring on DMSA and an abnormal ultrasound (S/A) by considering the other abnormalities found on DMSA. Of 52 S/N kidneys, 25 had other DMSA abnormalities. Of 40 S/A kidneys, 33 had other abnormalities ( $p=0.007 ; \chi^{2}$ test). If abnormal differential function was considered alone, as this may be considered a marker of objective renal damage, $14 \mathrm{~S} / \mathrm{N}$ kidneys had abnormal differential function, in contrast to 20 S/A kidneys $\left(p=0.023 ; \chi^{2}\right.$ test). Similarly the characteristics of kidneys where there were DMSA abnormalities but a normal ultrasound $(\mathrm{A} / \mathrm{N})$ were compared with those where there were DMSA abnormalities and an abnormal ultrasound (A/A) by considering the respective proportions where there was abnormal differential function on DMSA. Of $127 \mathrm{~A} / \mathrm{N}$ kidneys, 46 had abnormal differential function, whereas of $77 \mathrm{~A} / \mathrm{A}$ kidneys, 43 had abnormal differential function $\left(p=0.006 ; \chi^{2}\right.$ test).

\section{Discussion}

Ultrasound and DMSA imaging are the main diagnostic imaging investigations for childhood UTI in the UK. Despite the accepted low sensitivity of ultrasound for the detection of renal cortical scarring, surprisingly little data exists to quantify this. In infancy, when symptoms of UTI are non-specific and there is a greater risk of renal cortical scarring, ${ }^{13}$ there is little disagreement that imaging protocols should include ultrasound in the acute phase followed by static renal scintigraphy and micturating cystourethrography some weeks later, or earlier if there is a suspicion of obstructive uropathy. For children aged 1-5 years there is greater controversy, with current guidelines ${ }^{9}$ reflecting differing professional opinion on the need for DMSA imaging in this age group. ${ }^{14}$

We chose not to include data on cystography, as in practice this is a second line investigation outwith the infant age group. We have instead concentrated on the presence of renal parenchymal abnormalities, specifically cortical scarring, which is the end point that has been shown to be associated with an increased risk of hypertension and chronic renal insufficiency in later life. ${ }^{5}$

The BPA practice guidelines ${ }^{9}$ draw heavily from the data of Rickwood et al, who compared the ultrasound and intravenous urography findings in 200 children with UTI, and 
suggested that ultrasound alone was sufficient in children over the age of $2 .{ }^{14}$ However, their confidence in ultrasound is not universally shared, ${ }^{12} 15$ and their results are somewhat difficult to compare alongside the BPA guidelines as the children were grouped for analysis into $0-2$ and $2-10$ years of age. Other authors who have addressed the quantification of ultrasound sensitivity have been similarly beset by problems of small numbers, ${ }^{16}$ but our database of almost 1000 children has enabled us to have greater confidence in the reliability of our results, particularly when comparing children within different age groups.

Like Rickwood and colleagues, ${ }^{14}$ we found the prevalence of abnormalities to be high. We similarly found the sensitivity of ultrasound in detecting DMSA abnormalities or renal cortical scars to be low in all age groups, although there is an increase with age, significantly so $(p<0.05)$ between preschool and school aged children. Although sensitivity and specificity give information about the screening test, these figures do not take into account the prevalence of the condition. We have used information about ultrasound sensitivity and the prevalence of scarring to calculate the risk of missing scarring (the percentage of kidneys with normal ultrasound that have scarring detected on DMSA). We felt this provided a figure that would be clearly understood and applicable by both clinicians and health care economists. We also included calculations of risk for all DMSA abnormalities as well as DMSA cortical scarring, as there is no universal definition of renal cortical scarring ${ }^{11}$ and many of the additional abnormalities detected solely on DMSA imaging, such as focal photon deficiency or abnormal differential function, may be regarded as prescarring lesions. ${ }^{17}$ These results therefore represent an evaluation of the guidelines by substantiating clinical opinion with risk assessment data.

The risks have been calculated for individual kidneys but for practical purposes they may readily be translated to patient risks as the percentages of children with bilateral DMSA abnormalities and/or scarring were very small.

In order to make a valid comparison between the findings of the two imaging modalities, we set a time limit of 90 days difference between the date of the two procedures. In fact 768 of $990(78 \%)$ were carried out on the same day. It is known that changes seen on DMSA scans carried out soon after the UTI can be transitory. ${ }^{11}{ }^{12}$ Although the relevance of these changes remains unclear, in order to eliminate this variable, we excluded all patients who had a DMSA scan carried out within 30 days of the original clinical consultation date, as we felt this was a more objective date than an estimate of the date of the original UTI. Another reason for dating DMSA scans from the initial consultation rather than the date of the UTI was the fact that many children had had recurrent UTI by the time of the initial consultation. It was assumed that these children were more likely to have the UTI controlled, perhaps by prophylactic antibiotics after a first outpatient consultation, and therefore the time between first consultation and DMSA scanning represented the first infection free period.

Although any record in which the UTI clinical features were not clear was excluded from the database prior to analysis, it is possible that some patients were classified as having lower tract UTI who in fact had upper tract features. Similarly it is possible that a number of solitary UTIs were in fact recurrences. We recognise these as weaknesses of the retrospective design of the study but would suggest that such design limitations tend to minimise the ultrasound sensitivities and maximise the risks of missing scarring.

We compared the characteristics of the kidneys where DMSA abnormalities or scarring were detected by ultrasound and where it was missed. We found that where there was abnormal differential function, the ultrasound abnormality detection rate was significantly higher. This unsurprising result means that kidneys with more significant renal pathology at the time of scanning are detected more readily by ultrasound. However, there remains a substantial proportion of kidneys with abnormal differential function that are missed and we would also argue that as there is 17 -fold increase in the relative risk of progressive renal damage associated with scarring and subsequent documented infection, ${ }^{3}$ a scarred kidney represents a vulnerable kidney which merits close surveillance.

The BPA practice guidelines are generally endorsed by these risk assessments. Specifically we would advocate a universal DMSA imaging policy in all infants and all children with upper tract UTI, and we would suggest that in school age children with solitary lower tract UTI, the risk of missing scarring or DMSA abnormalities is acceptably low. In the preschool child with a solitary lower tract UTI there is a $1.2 \%$ risk of missing scarring (table 6) but a $4.9 \%$ risk of missing DMSA abnormalities (table 5). We anticipate that the former figure could be considered acceptable but that the latter may be more controversial. The figures for school age children with recurrent UTI are similar. The discrepancy between the risk of missing scarring and the risk of missing DMSA abnormalities highlights the need for follow up data in order to clarify the significance of abnormalities such as abnormal differential function and photon deficient areas without loss of cortex. The risks of missing DMSA abnormalities or scarring are higher for children with recurrent rather than solitary UTI in all categories except one and this highlights the importance of considering UTI recurrence separately in determining imaging protocols. The BPA guidelines do not consider recurrence separately from clinical features and we would suggest that for preschool children with recurrent lower tract UTI, the $2.5 \%$ risk of missing scarring would be unacceptable.

We would acknowledge that what is considered an acceptable risk needs to be debated by clinicians and health economists. We have provided figures in the form of a risk assessment where sparse objective data previously existed. Renal imaging policies are again the subject of 
debate $^{18}$ and it is hoped that this information will help in the formulation of future imaging protocols after childhood UTI.

The authors wish to acknowledge the computing assistance of Mr TM Muir; the help of the late Dr AS Hollman, and Dr AG Wilkinson and Dr BJ Fredericks, consultant paediatric radiologists; and the advice of Dr AV Murphy and Dr H Maxwell, consultant paediatric nephrologists; as well as the Renal Unit and Radiology Department secretarial staff and medical record staff at the Royal Hospital for Sick Children, Yorkhill, Glasgow.

1 Hellström A, Hanson E, Hansson S, Hjälmås K, Jodal U. Association between urinary sypmtoms at 7 years old and previous urinary tract infection. Arch Dis Child 1991;66:232-4.

2 Smellie JM, Hodson CJ, Edwards D, Normand ICS. Clini$\mathrm{cal}$ and radiological features of urinary infection in childhood. BMF 1964;2:1222-6.

3 Merrick MV, Notghi A, Chalmers N, Wilkinson AG, Uttley WS. Long-term follow up to determine the prognostic value of imaging after urinary tract infections. Part 2: scarring. Arch Dis Child 1995;72:393-6.

4 Merrick MV, Notghi A, Chalmers N, Wilkinson AG, Uttley WS. Long-term follow up to determine the prognostic value of imaging after urinary tract infections. Part 1 : reflux. Arch Dis Child 1995;72:388-92.

5 Jacobson SH, Eklöf AC, Eriksson CG, Lins L-E, Tidgren B, Winberg J. Development of hypertension and uraemia after pyelonephritis in childhood: 27 year follow up. BMf 1989 ; 299:703-6.

6 Martinell J, Jodal U, Lidin-Janson G. Pregnancies in women with and without renal scarring after urinary infection in childhood. BMF 1990;300:840-4.
7 Sacks SH, Verrier Jones K, Roberts R, Asscher AW, Ledingham JGG. Effect of symptomless bacteriuria in childhood on subsequent pregnancy. Lancet 1987;ii:991-4. 8 Guidelines for the management of acute urinary tract infection in childhood. $\mathcal{F}$ Coll Physicians Lond 1991;25:36-42.

9 Pilling D, Postlethwaite R. Imaging in urinary tract infection. London: British Paediatric Association, 1996.

10 Barry BP, Hall N, Cornford E, Broderick NJ, Somers JM, Rose DH. Improved ultrasound detection of renal scarring in children following urinary tract infection. Clin Radiol 1998;53:747-51.

11 MacKenzie JR. A review of renal scarring in children. Nucl Med Commun 1996;17:176-90.

12 MacKenzie JR, Fowler K, Hollman AS, Tappin DM, Murphy AV, Beattie TJ, Azmy AF. The value of ultrasound in the child with an acute urinary tract infection. $\mathrm{Br} \mathcal{F}$ Urol $1994 ; 74: 240-4$

13 Berg U, Johansson SB. Age as a main determinant of renal functional damage in urinary tract infection. Arch Dis Child 1983;58:963-9.

14 Rickwood AMK, Carty HM, McKendrick T, Williams MPL, Jackson M, Pilling DW, Sprigg A. Current imaging of childhood urinary infections: prospective study. $B M \mathcal{F}$ 1992;304:663-5.

15 Stokland E, Hellström M, Hansson S, Jodal U, Odén A, Jacobsson B. Reliability of ultrasonography in identification of reflux nephropathy in children. BMF 1994;309:235-9.

16 Smellie JM, Rigden SP, Prescod NP. Urinary tract infection: a comparison of four methods of investigation. Arch Dis Child 1995;72:247-50.

17 Majd M, Rushton HG. Renal cortical scintigraphy in the diagnosis of acute pyelonephritis. Semin Nucl Med 1992;2: 98-111.

18 Verrier Jones K. Prognosis for vesicoureteric reflux. Arch Dis Child 1999;81:287-94. 